\title{
Zunehmende Arbeitsmarktrisiken für Geringqualifizierte - Herausforderungen und Handlungsoptionen
}

Entgegen einer weit verbreiteten Auffassung sind die Erwerbsverläufe westdeutscher Beschäftigter in den letzten 25 Jahren nicht nachhaltig instabil geworden. $\mathrm{Zu}$ diesem Schluss kommen wir in einer aktuellen Studie. ${ }^{1}$ Auf eine Gruppe trifft diese Aussage allerdings nicht zu: Frauen und Männer ohne beruflichen Abschluss, die sogenannten Geringqualifizierten, wechseln heute deutlich häufiger den Arbeitgeber und sind zunehmend von Erwerbslosigkeit bedroht. Dieser nicht ganz unerwartete, in seiner Deutlichkeit aber doch überraschende Befund erscheint noch dramatischer, wenn man berücksichtigt, dass Deutschland in den letzten zehn bis 15 Jahren einen Anstieg der Lohnungleichheit erlebt hat, im Zuge dessen die Einkommen von Geringverdienern stagniert haben oder sogar zurückgegangen sind. Selbst wenn Geringqualifizierte also - was immer seltener der Fall ist - eine Beschäftigung finden, fällt das dadurch erzielte Einkommen häufig sehr niedrig aus.

Die materielle Lage von Personen ohne beruflichen Abschluss hat sich in den letzten Jahrzehnten also deutlich verschlechtert. Andersherum gesagt: Der individuelle, aber auch der gesellschaftliche Nutzen einer Berufsausbildung oder eines Studiums ist mittlerweile so hoch, dass umfassende Anstrengungen für eine Verbesserung der Bildungschancen nicht nur sozialpolitisch wünschenswert, sondern auch aus rein ökonomischer Sicht vernünftig sind. Eine Verbesserung der Bildungschancen lässt sich allerdings nur erreichen, wenn ein Kernproblem des deutschen Bildungssystems angegangen wird: die hohe soziale Vererbung von Bildung. In kaum einem anderen westlichen Land ist der Zusammenhang zwischen der Bildung der Eltern und der der Kinder so stark. Bei den Geringqualifizierten von morgen wird es sich also größtenteils um die Kinder der heutigen Geringqualifizierten handeln. Soll der Anteil der Geringqualifizierten verringert werden, geht es daher in erster Linie darum, die Bildungschancen von Kindern aus sogenannten bildungsfernen Familien zu verbessern. Zugleich müssen wir aber auch fragen, wie die Arbeitsmarktchancen von Personen erhöht werden können, die - aus welchen Gründen auch immer -, unzureichend ausgebildet sind und bleiben.

Vor diesem Hintergrund diskutieren wir im Folgenden wichtige Aspekte der genannten Probleme und skizzieren mögliche Lösungsansätze. Um den Beitrag zu strukturieren, orientieren wir uns an vier verschiedenen Phasen im Lebensverlauf. Im Hinblick auf das Vorschulalter plädieren wir für einen Perspektivwechsel, durch den vorschulische Betreuungseinrichtungen stärker als Bildungsinstitutionen begriffen und mit der entsprechenden finanziellen und personellen Ausstattung versehen werden. In Bezug auf das System der allgemeinbildenden Schulen thematisieren wir die Abwertung oder soziale Verarmung der Hauptschule, die eine völlige Abschaffung oder zumindest grundlegende Reform dieser Schulform nahelegt. Hinsichtlich der beruflichen Ausbildung und der daran anschließenden Phase der Erwerbs- und Familienarbeit plädieren wir unter anderem für eine Stärkung der vollzeitschulischen Ausbildung, für einen Ausbau der Möglichkeiten, Bildungsabschlüsse nachzuholen, sowie für eine Intensivierung der allgemeinen und betrieblichen Weiterbildung von Geringqualifizierten. Wir schließen mit den besonderen Problemen, denen sich Geringqualifizierte beim Übergang in den
Ruhestand gegenübersehen. Dabei wird deutlich, dass für diese Gruppe unter den gegenwärtigen Bedingungen ein drastischer Anstieg der Altersarmut zu erwarten ist.

\section{VORSCHULISCHE BILDUNG AUSBAUEN}

Die Erkenntnis ist nicht mehr neu: Keine Zeit ist so wichtig für die geistige und emotionale Entwicklung der Menschen wie die ersten Lebensjahre. Umso bedauernswerter ist es, dass die vorschulische Bildung in Deutschland lange Zeit stiefmütterlich behandelt wurde - insbesondere was die Betreuung von Kleinkindern zwischen einem und drei Jahren anbelangt. Hier sind erst in den letzten Jahren - auch und wohl sogar vorrangig mit dem Ziel, die Erwerbs-

\footnotetext{
Giesecke, J./Heisig, J. P. (2010): Destabilisierung und Destandardisierung, aber für wen? Die Entwicklung der westdeutschen Arbeitsplatzmobilität seit 1984, Kölner Zeitschrift für Soziologie und Sozialpsychologie 62 (3), S. 403-435.
}

Johannes Giesecke, Prof. Dr., ist Inhaber
des Lehrstuhls für Soziologie, insbesondere
Methoden der empirischen Sozialforschung,
an der Otto-Friedrich-Universität Bamberg.
Arbeitsschwerpunkte: Soziale Ungleichheit,
soziologische Arbeitsmarktforschung,
Methoden der empirischen Sozialforschung.
e-mail: johannes.giesecke@uni-bamberg.de
Jan Paul Heisig, Soziologe, ist
wissenschaftlicher Mitarbeiter in der
Abteilung Ungleichheit und soziale
Integration am Wissenschaftszentrum Berlin
(WZB). Arbeitsschwerpunkte: Vergleichende
Ungleichheits- und Arbeitsmarktforschung,
Zeitverwendungsforschung, quantitative
Methoden.
e-mail: heisig@wzb.eu


tätigkeit von jungen Müttern zu fördern verstärkte Anstrengungen unternommen worden. Die Förderung der Vereinbarkeit von Beruf und Familie ist unbestreitbar ein wichtiges gesellschaftspolitisches Ziel. Im Hinblick auf die Verbesserung der Bildungschancen geht es in erster Linie aber darum, den Bildungsauftrag der frühkindlichen Betreuung ernst zu nehmen. Dabei sollte es nicht darum gehen, einen vorschulischen, aber doch bereits schulähnlichen Unterricht einzurichten. Im Mittelpunkt der vorschulischen Bildung sollten vielmehr solche Anregungen stehen, die eine spielerische und zwanglose Aneignung der eigenen Umwelt fördern und die Entwicklung sozialer Kompetenzen begünstigen.

Den Bildungsauftrag der vorschulischen Betreuung ernst zu nehmen, bedeutet zunächst, größeres Augenmerk auf die Qualität der Kinderbetreuung zu legen. Vieles spricht dafür, dass hier beim gegenwärtigen Ausbau des Angebots in den alten Bundesländern allzu große Kompromisse gemacht werden. ${ }^{2}$ Vonnöten wären nicht zuletzt günstigere Betreuungsrelationen sowie eine verbesserte Ausbildung - und Bezahlung - der Erziehenden. Beides wird sich kaum erreichen lassen, ohne die Ausgaben für die frühkindliche Betreuung deutlich zu erhöhen. Folgt man den Berechnungen des amerikanischen Wirtschafts-Nobelpreisträgers James Heckman, wäre dieses Geld allerdings äußerst gewinnbringend angelegt. ${ }^{3}$

Ein weiteres Ziel muss sein, die Inanspruchnahme frühkindlicher Betreuung gerade unter den Geringqualifizierten zu erhöhen. Angesichts der zunehmenden Arbeitsmarktprobleme dieser Gruppe dürfte dies unter anderem erfordern, den Anspruch auf einen Krippenplatz weitgehend vom Vorliegen elterlicher Erwerbstätigkeit zu entkoppeln. Auch sollte der Besuch von Kinderkrippen für Familien mit niedrigem Einkommen kostenfrei oder z.B. aufgrund kostenloser Verpflegung sogar mit Einsparungen verbunden sein. In die falsche Richtung gehen hingegen steuerliche Instrumente wie das Ehegattensplitting oder Betreuungszuschüsse wie die sogenannte „Herdprämie“: Gerade für Geringqualifizierte, die oft nur geringe Erwerbseinkommen erzielen können, sind derartige direkte oder indirekte Zuschüsse klare Anreize, ihre Kinder selbst zu betreuen. Darüber hinaus bleiben sie in diesen Erziehungszeiten dem Arbeitsmarkt fern - mit den entsprechenden Folgepro- blemen für einen späteren Wiedereinstieg in Arbeit.

Langfristig könnte es zudem sinnvoll sein, auf eine vollständige Steuerfinanzierung vorschulischer Betreuungseinrichtungen umzustellen. Die Nutzung von Kinderkrippen und -gärten wäre dann wie die Nutzung allgemeinbildender Schulen komplett gebührenfrei. Bei progressiver Besteuerung würden Bezieher höherer Einkommen so automatisch stärker an der Finanzierung der frühkindlichen Bildung beteiligt. Auch Kinderlose würden in diesem Fall tendenziell einen größeren Finanzierungsbeitrag leisten als gegenwärtig - was angesichts der gesamtgesellschaftlichen Bedeutung der Kindererziehung aber durchaus erwünscht sein kann.

\section{MEHRGLIEDRIGES SCHULSYSTEM REFORMIEREN}

Ein charakteristisches Merkmal des deutschen Schulsystems ist eine frühe Ausdifferenzierung in verschiedene Schulzweige, die in der Regel nach der vierten, spätestens jedoch nach der sechsten Klasse erfolgt. Die Vor- und Nachteile des mehrgliedrigen Schulsystems und diesbezügliche Reformvorschläge sind immer wieder - wie zuletzt in Hamburg - Gegenstand heftiger Auseinandersetzungen. Aus wissenschaftlicher Sicht spricht vieles dafür, dass eine frühe Ausdifferenzierung Bildungschancen von Kindern aus bildungsfernen Elternhäusern verringert. ${ }^{4}$ Im deutschen Kontext ist hier eine Entwicklung besonders wichtig: Im Zuge der Bildungsexpansion ist es zu einer "Abwertung“ oder „sozialen Verarmung“ der Hauptschule gekommen. Die Schülerschaft der Hauptschulen rekrutiert sich - bei beträchtlichen regionalen Unterschieden vielerorts mittlerweile fast ausschließlich aus Elternhäusern mit niedrigem Bildungsniveau. Die Hauptschule ist zu einem Sammelbecken für diejenigen Schülerinnen und Schüler geworden, die es auch - aber eben längst nicht nur - infolge geringer Kompetenzen nicht auf die Realschule oder das Gymnasium geschafft haben. Es erscheint mehr als fraglich, ob Hauptschulen unter diesen Bedingungen noch förderliche Lernumwelten sein können.

Reformen sind hier unabdingbar, wenn die Bildungschancen benachteiligter Schüler erhöht werden sollen. Und wie bei der vorschulischen Bildung gilt: Deutschland wird die im internationalen Vergleich eher geringen Ausgaben für die schulische Bil- dung erhöhen müssen, da eine intensive und hochwertige Betreuung in der Regel eine Aufstockung des fachlich qualifizierten Personals erfordert. Nun werden sich allerdings nicht alle Probleme im Bildungssystem allein durch Mehrausgaben beseitigen lassen. Nötig ist sicherlich auch eine gesamtgesellschaftliche Debatte über Probleme des Bildungssystems und deren mögliche Lösungen. Wie das Beispiel Hamburg gezeigt hat, können Reformen im Schulsystem am Widerstand gut organisierter Teile des Bürgertums scheitern, wenn diese Gruppe davon überzeugt ist, dass die geplanten Veränderungen dem Wohl ihrer Kinder schaden.

\section{ZUGANG ZU AUS- UND WEITERBILDUNG FÖRDERN}

An die allgemeinbildende Schule schließt sich in Deutschland im Regelfall eine Berufsausbildung oder ein Hochschulstudium an. Das für Deutschland kennzeichnende System der dualen Ausbildung in Berufsschule und Betrieb kann in vielerlei Hinsicht nach wie vor als Erfolgsmodell gelten. Vielen Auszubildenden ermöglicht das duale System durch Praxisnähe und Anbindung an mögliche Arbeitgeber einen im internationalen Vergleich insgesamt eher reibungslosen Berufseinstieg. Eine Kehrseite des vergleichsweise standardisierten deutschen Ausbildungssystems ist, dass die Arbeitsmarktchancen stark von den Bildungsabschlüssen, d.h. den formal zertifizierten Qualifikationen, abhängen. So sind etwa die Arbeitsmarktrisiken von Personen ohne beruflichen Ausbildungsabschluss deutlich erhöht, selbst dann, wenn sie über einen Realschulabschluss oder gar ein Abitur verfügen.

Neben den bisher skizzierten Maßnahmen im (vor-)schulischen Bereich sind daher auch im Hinblick auf die eigentliche Berufsausbildung zusätzliche Anstrengungen erforderlich, damit die Zahl der Ausbildungslosen verringert wird. ${ }^{5}$ Hier zeigt

2 Vgl. Berth, F. (2010): Ausbildung zur Tagesmutter. Drei Tage Kurs müssen reichen, Süddeutsche Zeitung vom 26.10.

3 Heckman, J. (2006): Skill Formation and the Economics of Investing in Disadvantaged Children, in Science 312 (5782), S. 1900-1902.

4 Schütz, G./Ursprung, H./Wößmann, L. (2008): Education Policy and Equality of Opportunity, in: Kyklos 61 (2), S. 279-308.

5 Vgl. zum Folgenden auch Solga, H. (2009): Der Blick nach vorn: Herausforderungen an das deutsche Ausbildungssystem, WZB Discussion Paper SP I 507, Berlin. 
sich, dass vor allem Schulabbrecher und Hauptschulabsolventen, durchaus aber auch Realschulabgänger, immer größere Probleme haben, einen regulären Ausbildungsplatz zu erhalten - eine Tatsache, die von der offiziellen Statistik nicht erfasst wird, da diese nicht die wachsende Zahl von Jugendlichen in berufsvorbereitenden Maßnahmen („Übergangssystem“) berücksichtigt. Hier sollte unter anderem eine Stärkung vollzeitschulischer Ausbildungsgänge als Alternative zur dualen Ausbildung in Berufsschule und Betrieb erwogen werden. Angesichts einer beträchtlichen Zahl von Ausbildungsabbrüchen ist außerdem der Vorschlag bedenkenswert, die Transferierbarkeit von (Teil-) Leistungen und damit Wechsel zwischen Ausbildungsgängen zu erleichtern. Zusätzlich sollten die Rahmenbedingungen für einen nachholenden Erwerb allgemeinbildender und beruflicher Abschlüsse weiter verbessert werden, unter anderem durch einen Ausbau der Ausbildungsförderung oder die stärkere Berücksichtigung erhöhter Bedarfe (etwa aufgrund von Kindern) bei der Ausbildungsvergütung.

$\mathrm{Zu}$ fragen ist auch, inwieweit Arbeitgeber und Betriebe für verbesserte Ausbildungs- und Beschäftigungsperspektiven unter den Geringqualifizierten sorgen können. Wichtig ist in diesem Zusammenhang der Zugang zu betrieblichen Weiterbildungsangeboten. Für un- und angelernte Personen war dieser schon immer schwierig, die Zunahme von befristeter Beschäftigung sowie Leiharbeit haben diese Tendenz zuletzt aber noch deutlich verstärkt. Eine Vorbildfunktion könnte hier der öffentliche Dienst erfüllen, indem er durch gezielte Aus- und Fortbildungsangebote an Schulabbrecher und Hauptschüler deren Nachteile auf dem privaten Arbeitsmarkt kompensiert. Öffentliche Einrichtungen würden sich zudem hervorragend als Experimentierfelder für innovative Ansätze zur Förderung von Beschäftigten mit geringen formalen Qualifikationen eignen. Schließlich sollten Betriebsräte und Gewerkschaften darauf achten, dass die Chancen und Interessen Geringqualifizierter in der Bewerbungsphase und bei der Personalentwicklung besondere Berücksichtigung finden.

\section{ANSTIEG DER ALTERSARMUT ABWENDEN}

In der öffentlichen Debatte um die „Rente mit 67 " wurde zuletzt zunehmend die Frage aufgeworfen, ob die Erhöhung des Renteneintrittsalters angesichts anhaltend niedriger Beschäftigungsquoten bei den Über-60-Jährigen für die meisten Betroffenen nicht unweigerlich auf eine Rentenkürzung hinauslaufen werde. Die bisherige Diskussion ist begrüßenswert. Sie schärft den Blick dafür, dass die Verlängerung der Lebensarbeitszeit nicht einfach verordnet werden kann, sondern dass es dazu vielfältiger Voraussetzungen auf Seiten von Betrieben und Beschäftigten bedarf. Stärker als bisher sollte dabei berücksichtigt werden, dass verschiedene Personengruppen sehr unterschiedliche Beschäftigungschancen und gesundheitliche Voraussetzungen mit sich bringen. In dieser Hinsicht zeichnet sich eine Art sozialpolitischer Grundkonflikt dahingehend $a b$, dass die Geringqualifizierten Rentenkürzungen am wenigsten verkraften können, zugleich aber hohen Beschäftigungs- und Gesundheitsrisiken ausgesetzt sind und die Erwerbstätigkeit damit oft lange vor Erreichen des Renteneintrittsalters aufgeben müssen. Berücksichtigt man zudem die zunehmenden Erwerbsunterbrechungen in früheren Karrierephasen und die begrenzten finanziellen Spielräume für private Zusatzvorsorge, so erscheint ein massiver Anstieg der Altersarmut in dieser Gruppe beinahe unausweichlich.

Im Hinblick auf diese Probleme ist ein gesellschaftlicher Selbstverständigungsprozess dringend vonnöten. Sollte die gesetzliche Rentenversicherung vom sogenannten Äquivalenzprinzip abrücken und stärker redistributiv ausgerichtet werden? Müsste die Erhöhung des Renteneintrittsalters nicht von Veränderungen im Bereich der Erwerbsunfähigkeitsrenten flankiert werden? Müsste die Gesundheitsvorsorge nicht einen größeren Stellenwert in den Betrieben erhalten - und dabei sichergestellt werden, dass auch und gerade die Bedürfnisse geringqualifizierter Randbelegschaften Berücksichtigung finden? Es gibt gute Gründe, alle drei Fragen mit Ja zu beantworten. In jedem Fall sollte in der Öffentlichkeit und in den Betrieben stärker darüber diskutiert werden, wie der Übergang zur Rente mit 67 sozialverträglich ausgestaltet werden kann.

Gemeinsam ist den in diesem Beitrag skizzierten Vorschlägen, dass sie nicht zum Nulltarif zu haben sind. Zwar dürften sich vor allem die präventiven Investitionen im Bildungsbereich langfristig als gewinnbringend erweisen, kurzfristig aber würden sie in erster Linie auf der Ausgabenseite zu Buche schlagen. Sollen die Chancen von Geringqualifizierten verbessert und gleichzeitig dafür gesorgt werden, dass sich die Größe dieser Gruppe verringert, so bedarf es eines gesamtgesellschaftlichen Kraftakts. Leider ist ein solcher Kraftakt derzeit nicht in Sicht. Vielmehr herrschen Sparzwang, Kompetenzstreit und ideologische Grabenkämpfe vor. Die deutsche Gesellschaft muss sich dennoch fragen lassen, ob sie es mit ihren Grundprinzipien vereinbaren kann, dass sich die Lebenschancen und -perspektiven eines größeren Teils der Bevölkerung zunehmend verschlechtern. 